\title{
Modificación de los aceros rápidos de herramientas AISI M2 por fusión superficial con láser bajo diferentes condiciones de operación ${ }^{(\cdot)}$
}

\author{
J. Arias ${ }^{*}$, M. Cabeza* ${ }^{* *}$, G. Castro ${ }^{*}$, I. Feijoo ${ }^{* *}$, P. Merino** y G. Pena ${ }^{* *}$
}

Resumen

\begin{abstract}
Se ha aplicado el tratamiento superficial de fusión con láser a un acero comercial rápido de herramientas AISI M2, templado y revenido, y se ha estudiado su microestructura superficial y comportamiento mecánico (dureza y resistencia al desgaste). Para ello, se ha empleado un láser continuo Nd:YAG, variando las condiciones de operación, analizando la influencia de los distintos parámetros sobre cordones individuales y sobre las características de la superficie fundida cuando se realiza el tratamiento en multipasada con un solape del $50 \%$. La caracterización microestructural realizada de la superficie fundida muestra que, en todos los casos, se encuentra constituida por carburos de pequeño tamaño, $\mathrm{M}_{2} \mathrm{C}$ y $\mathrm{MC}$, martensita y austenita retenida, cuya cantidad depende de los parámetros de operación. Así mismo, se ha determinado que valores bajos de densidad de potencia y altas velocidades de barrido del haz conducen a mayor homogeneidad en la microestructura, con altos valores de dureza y resistencia al desgaste.
\end{abstract}

Palabras clave $\quad$ Acero rápido AISI M2; Láser Nd:YAG; Fusión superficial por láser; Dureza; Resistencia al desgaste.

\section{Modification of AISI M2 high speed tool steels after laser surface melting under different operation conditions}

\begin{abstract}
We applied a laser surface melting treatment to AISI M2 high-speed steel -hardened and tempered-and studied the resulting surface characteristics (microstructure) and mechanical behavior (hardness and wear performance). The steel was treated using a Nd:YAG continuous-wave laser with different operation conditions. The influence of the laser processing parameters on the single tracks and on melted surface layer obtained by multipass system with $50 \%$ overlap were studied. The microstructure for all conditions is formed by $\mathrm{MC}$ - and $\mathrm{M}_{2} \mathrm{C}$-type carbides, martensite and retained austenite; the quantities of this phase depends on the operations conditions. It has been determined that low levels of power density and high speed scanning of the beam leads to greater homogeneity in the microstructure with high hardness values and wear resistance.
\end{abstract}

Keywords

AISI M2 high speed steel; Nd:YAG laser; Laser melting; Hardness; Wear.

\section{INTRODUCCIÓN}

Los aceros rápidos pertenecen al grupo de los aceros de herramientas y se caracterizan por estar altamente aleados. En general, contienen grandes cantidades de wolframio y/o molibdeno junto con cromo, vanadio o cobalto ${ }^{[1]}$.

El acero rápido objeto de estudio es un AISI M2. Generalmente, se emplea en la fabricación de herramientas de corte que son utilizadas en operaciones de mecanizado en tornos, sierras, limadoras, fresadoras, brocas, etc., pero su uso también se extiende, de forma creciente, a la fabricación de troqueles de conformado, debido a que posee una combinación de buena tenacidad y resistencia. En estas operaciones, la superficie del acero está sometida a condiciones de desgaste, compresión y altas temperaturas, de modo que las propiedades de la misma en contacto con la pieza de trabajo, jugarán un papel fundamental en la vida útil de las herramientas. Por ello, se acepta que las medidas

\footnotetext{
(•) Trabajo recibido el día 31 de marzo de 2009 y aceptado en su forma final el día 12 de enero de 2010.

Centro Tecnológico (AIMEN). Relva 27 A. Torneiros.36410. Porriño, Pontevedra, España.

** Departamento de Ingeniería de los Materiales, Mecánica Aplicada y Construcción. E.T.S.I. Industriales. Universidad de Vigo. Campus de Lagoas-Marcosende.36310, Vigo, España.
} 
de dureza y resistencia al desgaste son adecuadas para estimar el comportamiento de estos materiales en condiciones de servicio, y los tratamientos propuestos para mejorar su durabilidad, deben promover elevados valores de estas propiedades ${ }^{[1 \text { y } 2] \text {. }}$

El comportamiento superficial puede mejorarse por diversos métodos y, en los últimos años, junto con los tradicionales (nitruración, deposición física en fase vapor-PVD- y deposición química en fase vapor-CVD-), se está utilizando el láser en los tratamientos superficiales. El empleo de esta nueva técnica tiene una serie de ventajas como son, entre otras: control exacto de la zona a tratar así como su profundidad, además puede ser aplicado a piezas con formas complejas y es un sistema rápido y de fácil automatización ${ }^{[3]}$.

Las posibilidades del tratamiento superficial de los aceros de herramientas empleando la tecnología láser son varias, que van desde un calentamiento superficial suficiente como para producir un cambio microestructural a la fusión y su posible aleación con otros elementos o incluso producir recubrimientos. Todos estos posibles tratamientos y su efecto sobre el comportamiento final de los aceros de herramientas lleva siendo objeto de investigación desde hace algunos años. Así, trabajos recientes muestran cómo el temple superficial empleando la tecnología láser sobre aceros de herramientas de medio carbono aumenta la resistencia a desgaste y corrosión ${ }^{[4]}$. La mejora en el comportamiento tribológico, empleando este endurecimiento superficial por temple con láser, igualmente tiene lugar cuando el acero tiene alto contenido en carbono ${ }^{[5]}$. También, el recubrimiento superficial de aceros para moldes con aceros rápidos de herramientas empleando la tecnología láser ha resultado un buen método para mejorar su resistencia al desgaste ${ }^{[6]}$.

Una de las aplicaciones que más interés suscita es la fusión superficial por láser pues en el caso de los aceros de herramientas permite obtener estructuras en la superficie que presentan gran dureza y que tienen buen comportamiento a desgaste $\mathrm{e}^{[7}$ y 8$]$ y a la corrosión ${ }^{[9]}$. En el tratamiento se emplea un haz láser de alta potencia para barrer la superficie, de modo que se eleva la temperatura del metal por encima de la de fusión. La rápida fusión de la superficie que tiene lugar, seguida de una, también rápida, solidificación del metal fundido, modifica la distribución de los elementos de aleación y la microestructura de la zona tratada. La fusión superficial por láser produce la disolución de las largas cadenas de carburos típicas de estos aceros en la condición de forjados, el afino de la microestructura y la homogenización química ${ }^{[10]}$, que pueden conducir a una mejora de la resistencia al desgaste. La microestructura final depende en gran medida de la velocidad de enfriamiento de la superficie fundida que, con este tratamiento, puede alcanzar valores de hasta $10^{6} \mathrm{~K} / \mathrm{s}$, muy superiores a los $10^{2}$ $\mathrm{K} / \mathrm{s}$ o inferiores, que se obtienen en una solidificación convencional ${ }^{[11]}$.

En los últimos años se han publicado diversos trabajos en los que se estudia la influencia de los parámetros de operación del láser en la formación de microestructuras durante la solidificación de este acero rápido $\mathrm{M}^{[12-16]}$. Sin embargo, los resultados obtenidos en estos estudios no son coincidentes y las diferencias se pueden atribuir al tipo de láser empleado, la densidad de potencia y la velocidad de desplazamiento del haz de láser. Hay que señalar, además, que estos estudios se han realizado sobre cordones individuales y cuando se quiere tratar una superficie completa es necesario realizar la fusión superficial en pasadas sucesivas con un determinado grado de solapamiento que puede producir variaciones en la microestructura de los cordones individuales.

Por otro lado, las microestructuras de solidificación rápida del acero rápido de herramientas $\mathrm{M} 2$ a temperatura ambiente presentan proporciones variables de austenita retenida ${ }^{[17]}$, fase inestable en servicio que puede reducir las mejoras producidas por la fusión por láser. Este constituyente no es identificado en todos los casos ya estudiados por otros autores ${ }^{[12-15]} \mathrm{y}$ quien lo indica, señala que las cantidades son pequeñas, sin cuantificación. Su presencia y cantidad parece estar asociada a los parámetros de operación de láser, como han sugerido otros autores ${ }^{[18 \text { y } 19]}$ que han realizado estudios sobre un acero de herramientas inoxidable (AISI 420) con menor contenido en carbono que el AISI M2. La eliminación de este constituyente pasa por la realización de temples sucesivos en la pieza tratada superficialmente ${ }^{[20 \text { y } 21]}$ tras austenización completa, integrando el tratamiento superficial en el proceso de fabricación de la herramienta. Sin embargo esto no es posible cuando la fusión por láser se realiza sobre piezas finales o con el objeto de aumentar la vida útil de herramientas dañadas en servicio.

Por ello, en el presente trabajo se ha tratado por fusión superficial, empleando un láser de haz continuo $\mathrm{Nd}$ :YAG, el acero rápido AISI M2, variando las condiciones de operación con objeto de establecer una relación clara entre estos parámetros y la microestructura obtenida en cordones individuales. Así mismo, se han tratado superficies de este material empleando las condiciones anteriores con solapamiento del $50 \%$, estudiando, en todos los casos, la microestructura y las propiedades mecánicas (dureza y resistencia al desgaste). El propósito de este trabajo es, además de estudiar la posibilidad de reducir a mínimos la austenita retenida, obtenida tras tratamiento de fusión por láser en el caso de este acero de herramientas, 
analizar si su presencia resulta determinante en las propiedades mecánicas de la superficie (dureza y resistencia a desgaste). También, se valora si el sistema de tratamiento superficial empleando el solapamiento induce heterogeneidades importantes en la respuesta mecánica de la superficie.

\section{MATERIALES Y PROCEDIMIENTO EXPERIMENTAL}

\subsection{Material utilizado}

Se ha utilizado un acero forjado rápido de herramientas AISI M2, recibido en estado de recocido y que, posteriormente, fue austenizado a $1.210^{\circ} \mathrm{C}$, templado en aceite y revenido tres veces a $565^{\circ} \mathrm{C}$ durante $2 \mathrm{~h}$ a la temperatura de tratamiento de cada ciclo térmico, con objeto de optimizar la tenacidad para la dureza conseguida, según las recomendaciones del fabricante de estos aceros.

La composición química del acero se muestra en la tabla I.

\subsection{Procedimiento experimental}

Los tratamientos de fusión superficial por láser se han realizado en probetas rectangulares de dimensiones 150 x $100 \mathrm{~mm}^{2}$ y $15 \mathrm{~mm}$ de espesor. Se ha empleado un láser continuo de Nd:YAG, marca Rofin, modelo DY044, bombeado por diodos de 4,4 kW de potencia máxima, emitiendo un haz de distribución multimodo y de distribución de potencia gaussiana para obtener una distribución espacial de la densidad de potencia en la superficie lo más homogénea posible. Se utilizó una fibra óptica de $400 \mathrm{~mm}$ de diámetro, del tipo MMI, para llevar el haz hasta la superficie de la probeta.

Se realizaron cordones simples, obtenidos mediante una sola pasada del haz láser sobre las muestras M21, M22, M23 y M24. Los parámetros de operación empleados en cada uno de los cordones pueden verse en la tabla II. Todos ellos se obtuvieron aplicando la misma potencia (P) de $2 \mathrm{~kW}$ y la misma relación distancia focal/diámetro del haz (6), variando la velocidad de desplazamiento del haz (v) y la distancia focal, variando de ese modo el diámetro del haz $\left(\varnothing_{\text {haz }}\right)$ incidente sobre la superficie del metal. Como consecuencia, se obtuvieron distintas densidades de potencia y energías aportadas por unidad de superficie del haz láser en cada cordón fundido. En todos los casos, con objeto de recubrir la superficie de estudio, se realizó el tratamiento de fusión superficial haciendo pasadas múltiples con un solapamiento entre ellas del $50 \%$.

Las probetas fundidas superficialmente se caracterizaron microestructural y mecánicamente.

La caracterización microestructural se ha realizado empleando microscopía óptica y electrónica, así como difracción de rayos X (DRX). Una vez desbastadas y pulidas las secciones transversales de las muestras, tras

Tabla I. Composición química del acero AISI M2 (\% en peso)

Table I. Chemical composition for the AISI M2 steel (weight \%)

\begin{tabular}{ccccccccc}
\hline $\mathbf{C}$ & $\mathbf{M n}$ & $\mathbf{P}$ & $\mathbf{S}$ & $\mathbf{S i}$ & $\mathbf{C r}$ & $\mathbf{W}$ & Mo & $\mathbf{V}$ \\
\hline 0,93 & 0,27 & 0,043 & $<0,009$ & 0,33 & 4,15 & 6,19 & 4,62 & 1,83 \\
\hline
\end{tabular}

Tabla II. Parámetros de operación del láser Nd: YAG

Table II. Nd:YAG laser operational parameters

\begin{tabular}{|c|c|c|c|c|c|}
\hline Cordón & $\begin{array}{c}P \\
(k W)\end{array}$ & $\begin{array}{c}v \\
(\mathrm{~mm} / \mathrm{s})\end{array}$ & $\begin{array}{c}\varnothing_{\text {haz incidente }} \\
(\mathrm{mm})\end{array}$ & $\begin{array}{c}\text { Densidad de } \\
\text { potencia }\left(\mathrm{W} / \mathrm{mm}^{2}\right)\end{array}$ & $\begin{array}{c}\text { Energía aportada } \\
\left(\mathrm{J} / \mathrm{mm}^{2}\right)\end{array}$ \\
\hline M21 & 2 & 25 & 3,33 & 229,69 & 24,06 \\
\hline M22 & 2 & 25 & 6 & 70,73 & 13,33 \\
\hline M23 & 2 & 60 & 3,33 & 229,64 & 10 \\
\hline M24 & 2 & 60 & 6 & 70,73 & 5,55 \\
\hline
\end{tabular}


su ataque con el reactivo Vilella, se han observado en el microscopio óptico Olympus, modelo PMG3, y el electrónico de barrido, modelo JSM-5410. Los constituyentes del acero antes y después del tratamiento de fusión con láser se han estudiado empleando DRX. Los espectros de DRX se obtuvieron en un difractómetro Siemens D5000 con radiación Cu Ka $(\lambda=1,418 \AA)$. Los ensayos se realizaron entre los ángulos de barrido $30^{\circ} \leq 2 \theta \leq 100^{\circ}$, con paso de $0,02^{\circ}$, y $10 \mathrm{~s}$ de tiempo de permanencia por paso. Los picos se identificaron con el programa EVA haciendo uso de los patrones estándar de difracción (ICCD). Los parámetros de la celda unidad de los carburos se calcularon con el programa. WIN metric. El análisis de la austenita retenida se ha realizado de acuerdo a la norma E 975-03, "Standard Practice for X-Ray Determination of Retained Austenite in Steel with Near Random Crytallographic Orientation". Las medidas se han realizado en la superficie del material tras tratamiento de fusión con láser con multipasada, que han sido rectificadas mínimamente (a una altura que no sobrepase la zona fundida), lo que es necesario pues la norma E 975-03 indica que la medida ha de realizarse sobre una superficie pulida a $6 \mu \mathrm{m}$. De todos modos, una vez realizado el ensayo se han atacado y observado al microscopio para constatar que se trata de la zona fundida.

Tal como se indicó anteriormente, la caracterización mecánica (dureza y resistencia al desgaste) se realizó en la superficie tratada, que será la que tenga que soportar en servicio las condiciones de trabajo.

Al realizar el tratamiento de fusión por láser de la superficie empleando solapamiento del $50 \%$ se produce la fusión de parte del material previamente fundido y el calentamiento de la zona contigua. Esto producirá modificaciones microestructurales que se traducirán en variaciones de dureza. Para detectarlo, se han realizado perfiles de $\mathrm{HV}_{0,5}$, perpendiculares al avance de cordón en la superficie tratada, según la norma ASTM 384-89: "Standard Test Method for Microindentation Hardness of Materials". Así mismo, se ha determinado la microdureza media de la superficie fundida para cada una de las condiciones de operación.

La resistencia al desgaste por deslizamiento se evaluó en todos los casos mediante ensayos pin-on-disk, según la norma ASTM G99 "Standard test method for wear testing with a pin-on-disk apparatus". Se realizaron ensayos a temperatura ambiente, empleando una carga de $10 \mathrm{~N}$ y una bola de alúmina de $3 \mathrm{~mm}$ de diámetro, a una velocidad de giro de 213 r.p.m. El radio de giro fue de $4,5 \mathrm{~mm}$ y la distancia de deslizamiento de $1.000 \mathrm{~m}$. Para medir el volumen perdido durante el ensayo, se empleó un perfilómetro mecánico. Las huellas de desgaste se observaron al microscopio electrónico de barrido (MEB).

\section{RESULTADOS Y DISCUSIÓN}

El acero rápido AISI M2, tras tratamiento térmico convencional presenta una microestructura de martensita con carburos de diferente morfología y gran tamaño alineados en bandas paralelas a la dirección de forja, como puede verse en la figura 1 . Tras el tratamiento térmico de fusión por láser tienen lugar cambios microestructurales en la superficie tratada. La figura 2 muestra la sección transversal de los cordones simples obtenidos empleando los parámetros de operación del láser ensayados (Tabla II). Para todas las condiciones consideradas, los cordones presentan una ausencia de defectos como fisuras, poros o microrechupes, lo que nos indica que todos los parámetros de procesado láser utilizados son los adecuados para obtener una superficie libre de defectos.

Se puede destacar, de la observación de la figura 2, la influencia que tienen los parámetros de operación del láser sobre la morfología de los cordones. Al aumentar la energía aportada por unidad de superficie aumentan, significativamente, las dimensiones de la zona afectada por el láser. La tabla III muestra la profundidad total obtenida en la parte central de los cordones simples de cada una de las muestras.

Para todas las condiciones consideradas, en la sección transversal pueden observarse las distintas zonas características de este tratamiento superficial ${ }^{[20]}$ (Fig. 3): la zona fundida (ZF) donde se produjo la fusión completa del material, la zona afectada por el calor (ZAC) donde el material se llegó a calentar

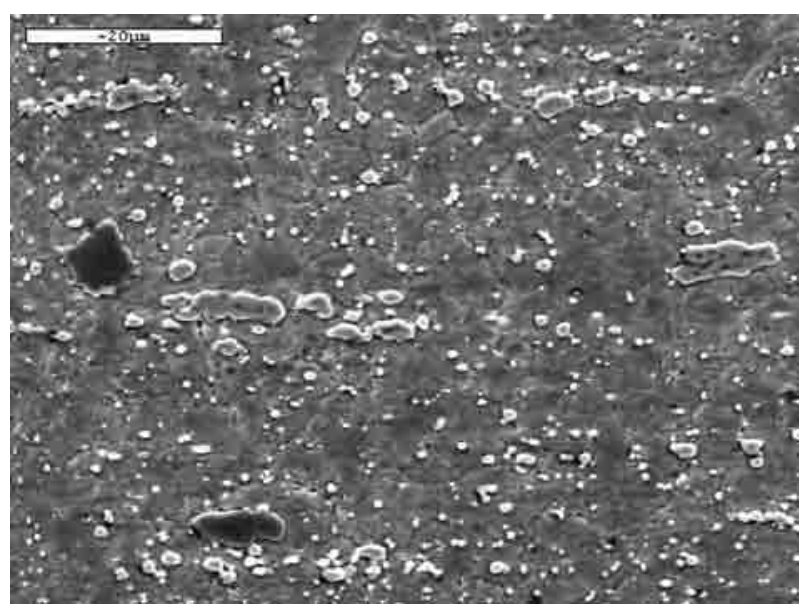

Figura 1. Micrografía, obtenida al MEB, de la sección transversal de una muestra del acero AISI M2, templado y revenido.

Figure 1. SEM micrograph of cross-section of a hardened and tempered AISI M2 high speed steel sample. 


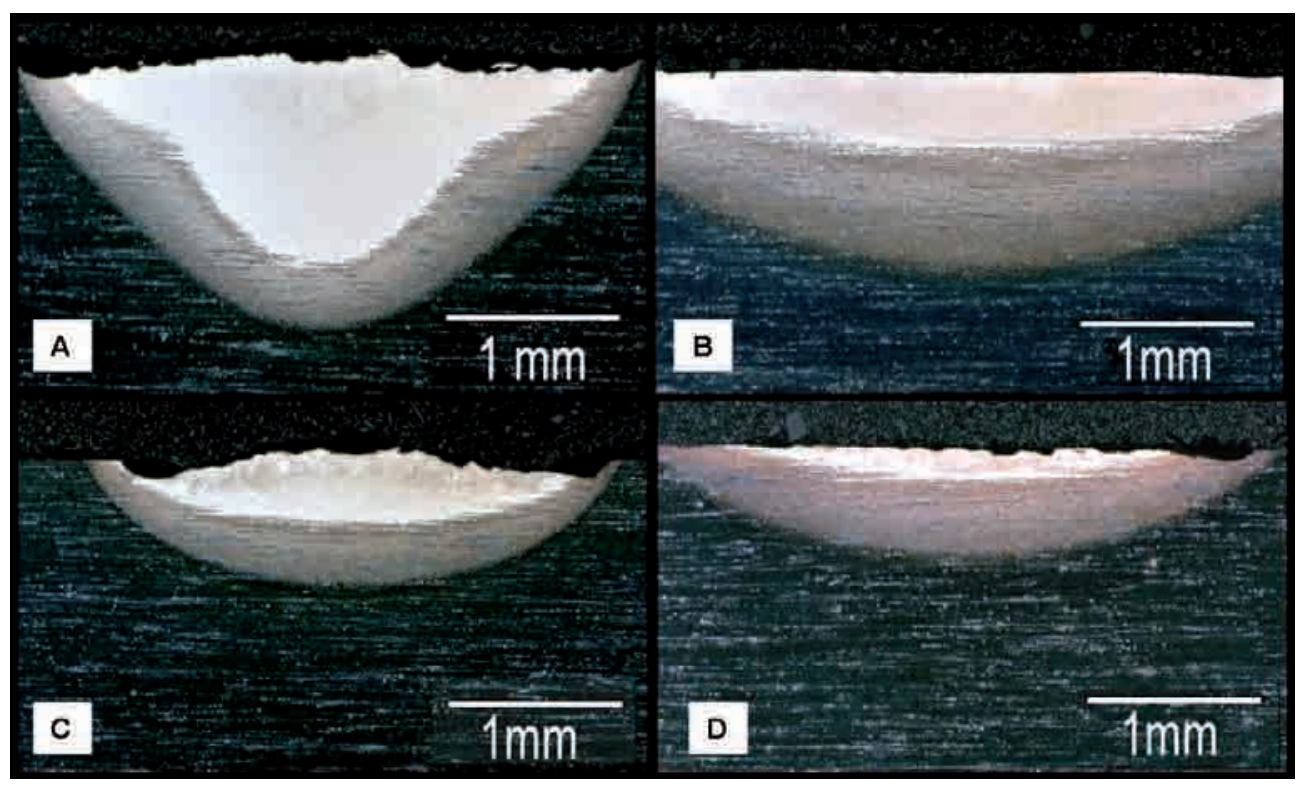

Figura 2. Sección transversal de los cordones. Reactivo de ataque Vilella: A) Cordón M21. B) Cordón M22. C) Cordón M23. D) Cordón M24.

Figure 2. Cross-section of samples. Etchant Vilella: A) Sample M21. B) Sample M22. C) Sample M23. D) Sample M24.

Tabla III. Espesor de las zonas observadas en las sección transversal de los cordones simples

Table III. Thickness of the observed zones in the cross section of the single laser pass

\begin{tabular}{crrrr}
\hline Zona & \multicolumn{1}{c}{ M21 } & M22 & \multicolumn{1}{c}{ M23 } & M24 \\
\hline MF & $968 \mu \mathrm{m}$ & $330 \mu \mathrm{m}$ & $308 \mu \mathrm{m}$ & $84 \mu \mathrm{m}$ \\
ZPF & $43 \mu \mathrm{m}$ & $132 \mu \mathrm{m}$ & $51 \mu \mathrm{m}$ & $70 \mu \mathrm{m}$ \\
ZAC & $281 \mu \mathrm{m}$ & $330 \mu \mathrm{m}$ & $206 \mu \mathrm{m}$ & $213 \mu \mathrm{m}$ \\
Total & $1292 \mu \mathrm{m}$ & $853 \mu \mathrm{m}$ & $566 \mu \mathrm{m}$ & $368 \mu \mathrm{m}$
\end{tabular}

pero en ningún caso se fundió, la zona parcialmente fundida $(\mathrm{ZPF})$, que es la zona de transición entre la zona fundida y la afectada por el calor, y el material base (MB) que no ha sido afectado por el tratamiento de fusión superficial. Cada una de ellas, ha sido caracterizada microestructuralmente en estudios anteriores $^{[22]}$, determinando la microestructura de la matriz y el tipo y la distribución de carburos precipitados. El resultado muestra que cada una de las zonas tiene una microestructura distinta, consecuencia de la temperatura alcanzada en la fusión y del régimen de enfriamiento. Así, en la ZPF la temperatura no ha sido suficientemente elevada para producir la

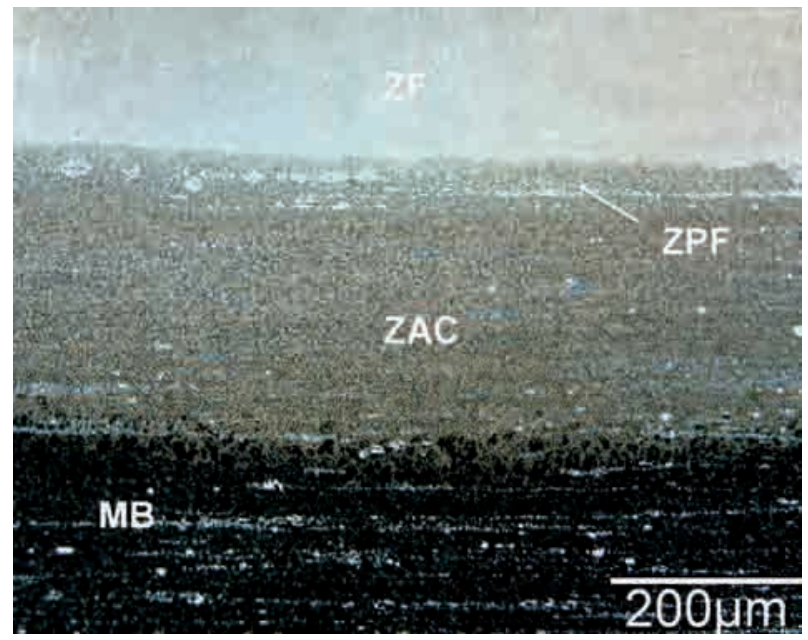

Figura 3. Zonas características del tratamiento de fusión con láser. Reactivo de ataque Vilella.

Figure 3. Characteristic zones of the treatment melting laser. Etchant Vilella.

completa disolución de los carburos masivos y, como puede verse en las figuras $4 \mathrm{~A}$ y $4 \mathrm{~B}$, presenta una microestructura heterogénea consecuencia del tamaño de los mismos; es previsible que si, en el acero de partida, los carburos primarios se encontraran más homogéneamente distribuidos en la matriz y de menor 


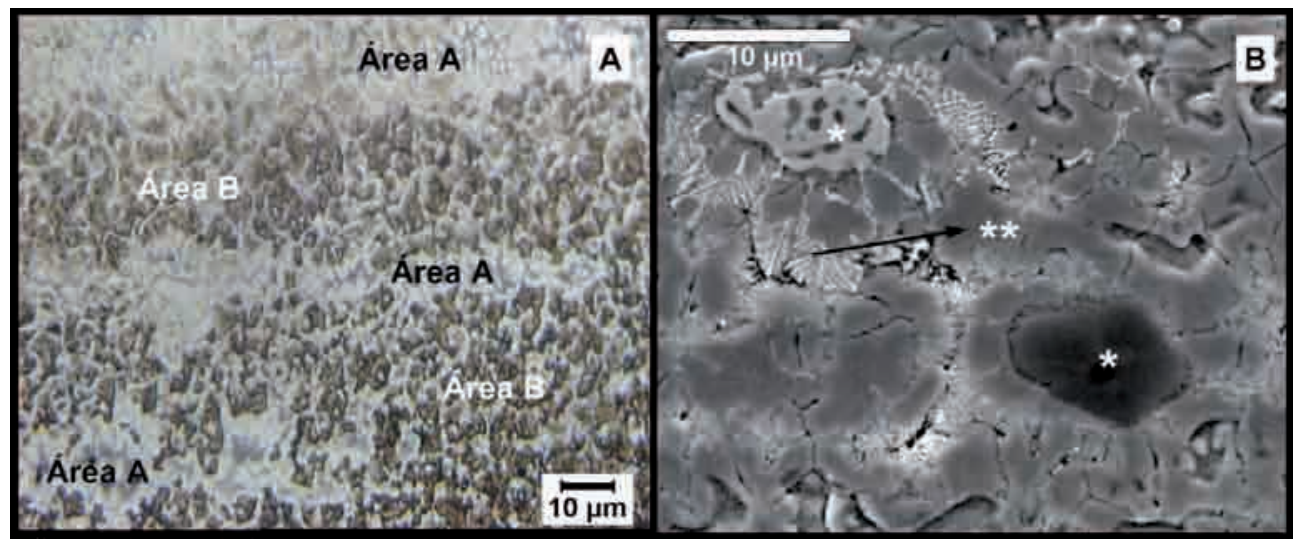

Figura 4. Sección transversal: zona parcialmente fundida (ZPF). Reactivo de ataque Vilella. A) Micrografía óptica donde se observa la heterogeneidad de la zona - área A y área B-. B) Micrografía del MEB del área A que se encuentra asociada a carburos primarios masivos no disueltos $\left({ }^{*}\right)$ rodeados de una estructura de solidificación rápida con carburos eutécticos del tipo espina de pescado $\left.{ }^{* *}\right)$.

Figure 4. Cross-section: partial melted zone (PMZ). Etchant Vilella. A) Optical micrograph showing the heterogeneity of the zone $-A$ area and $B$ area. $B$ ) SEM image showing the $A$ area that is associated to massive no dissolved primary carbides $\left(^{*}\right)$ surrounded by a structure of rapid solidification with fishbone eutectic carbides.

tamaño, la ZPF sería más homogénea y estrecha. Por otro lado, la ZF, que es la que estará en contacto directo con el material a conformar y sometida a desgaste durante el servicio de la herramienta, tiene una microestructura típica de solidificación de este tipo de aceros pero con dendritas de pequeño tamaño $(1-2 \mu \mathrm{m})^{[5]}$, como ilustran las figuras $5 \mathrm{~A}$ y $5 \mathrm{~B}$. Dichas dendritas, que nacen a partir de granos columnares, aparecen rodeadas de una eutéctica de austenita (que, a temperatura ambiente, se habrá transformado en martensita, oscurecida por el reactivo Vilella) y carburos $\mathrm{M}_{2} \mathrm{C}^{[22]}$, consecuencia de la reacción eutéctica $\left(\mathrm{L} \rightarrow \mathrm{A}+\mathrm{M}_{2} \mathrm{C}\right)$ sufrida por el último líquido en solidificar. La presencia de carburos del tipo $\mathrm{M}_{2} \mathrm{C}$ está

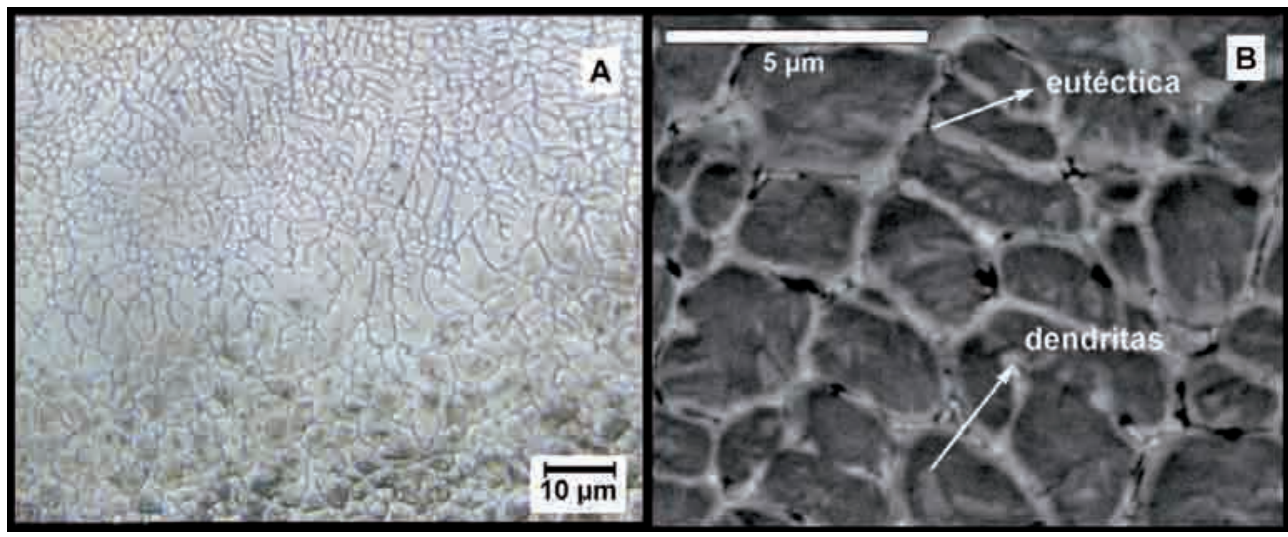

Figura 5. Sección transversal: zona fundida (ZF). Reactivo de ataque Vilella. A) Micrografía óptica. B) Micrografía del MEB.

Figure 5: Cross-section: melted zone (MZ). Etchant Vilella. A) Optical micrograph; B) SEM image. 
justificada por la rápida velocidad de enfriamiento desde la temperatura de fusión, que favorece la formación de estos carburos ${ }^{[17]}$.

En la tabla III se muestra que el espesor de cada una de las zonas depende de los parámetros de operación: densidad de potencia y velocidad de barrido. Para densidad de potencia constante se produce una reducción en la profundidad de la zona fundida cuando aumenta la velocidad del haz del láser (de $25 \mathrm{~mm} / \mathrm{s}$ a $60 \mathrm{~mm} / \mathrm{s}$ ). Este efecto, que ya ha sido observado por varios autores $^{[7,8 \text { y } 13]}$, se debe a una disminución del calor impuesto por unidad de área sobre la superficie, al reducirse el tiempo de interacción sobre la misma. Resulta destacable que para velocidad del haz de láser constante se produce una disminución de la ZPF al disminuir el diámetro del haz incidente. La explicación de este hecho tiene su origen en la naturaleza de la ZPF, que es aquella en que no se ha alcanzado la fusión completa del acero y está ligada a la presencia de carburos masivos difíciles de disolver cuando se encuentran a una cierta profundidad pues no les llega energía suficiente. La disminución del diámetro del haz incidente (de $6 \mathrm{~mm}$ a 3,33 $\mathrm{mm}$ ) ha tenido lugar por aproximación del foco del haz del láser sobre la superficie, de tal manera que aumenta la densidad de potencia hasta el punto que se puede a llegar a vaporizar el metal debajo del centro del haz ${ }^{[3]}$. La presión de expansión del vapor mantiene la cavidad abierta formando un cuerpo negro que transfiere más energía al interior, siendo posible fundir carburos masivos en zonas que, con menor densidad de potencia, no es posible.

Para cubrir totalmente la superficie de trabajo es necesario solapar cordones individuales. El resultado de la operación para cada una de las condiciones puede observarse en la figura 6, en la que se ven las micrografías de la sección transversal del material tras el tratamiento de fusión por láser. En todos los casos, se observa ausencia de defectos y la microestructura en la ZF no varía. En dicha figura pueden observarse, en las distintas secciones transversales, las mismas zonas que se obtenían con los cordones individuales y su espesor se ha visto afectado de la misma manera por los parámetros de operación del láser. Es interesante señalar que cuando la densidad de potencia del haz del láser no es elevada (M22 y M24) la superficie que se obtiene parece que tiene menor rugosidad, factor a tener en cuenta si el tratamiento se va a emplear para reparar piezas.

Tras el tratamiento de fusión superficial multipasada en la superficie se puede distinguir en cada cordón producido por el paso del haz del láser dos zonas, una ZF y una ZAC consecuencia del calor cedido por el haz del láser al material adyacente (Fig. 7). No hay ZPF porque, en la superficie, el haz del láser transmite su energía sobre material que ha sido fundido previamente y tiene una microestructura con pequeños carburos más fácil de fundir que los carburos masivos de acero rápido convencional. En la figura 7D, en la que se muestran las dos zonas separadas por una banda clara que corresponde al comienzo de la ZF (granos equiáxicos), puede verse que, en todo caso, la microestructura es dendrítica, no apreciándose diferencias sustanciales. Al calentar a elevadas temperaturas, sin llegar a fundir, podría producirse un cierto revenido del acero con modificación de la microdureza, si el tiempo de permanencia es suficiente. Sobre las bandas, en todos los casos, se ha medido la microdureza y los valores medios aparecen en la figura 8 . Puede decirse que, en general, la variación

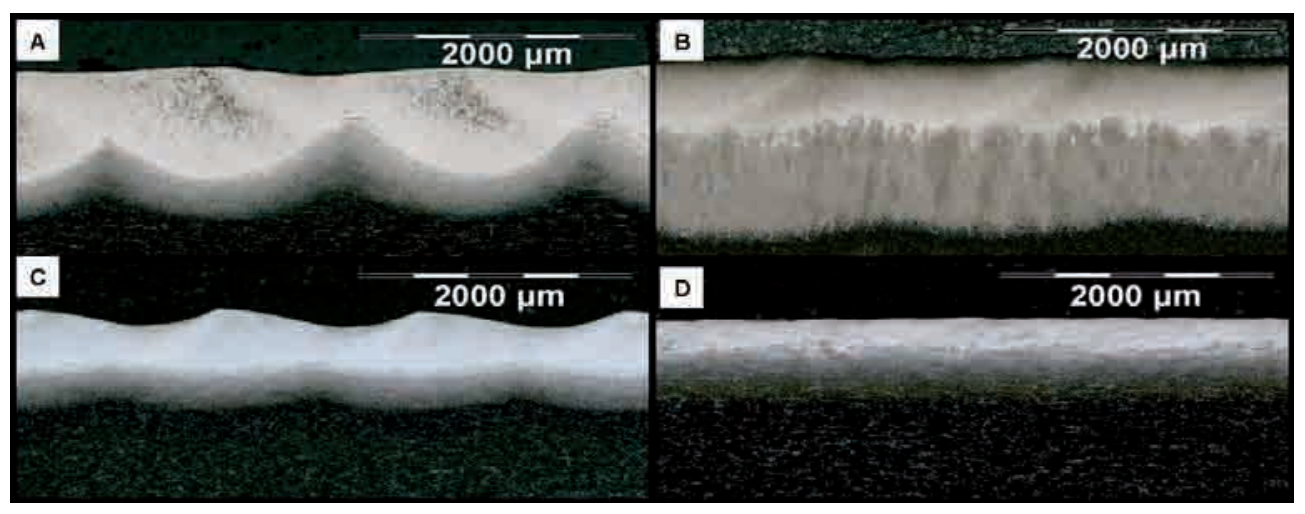

Figura 6. Sección transversal: solapes al $50 \%$. Reactivo de ataque Vilella: A) M21. (B) M22. C) M23. D) M24.

Figure 6. Cross-section: 50 \% overlap. Etchant Vilella: A) M21; B) M22; C) M23: D) M24. 


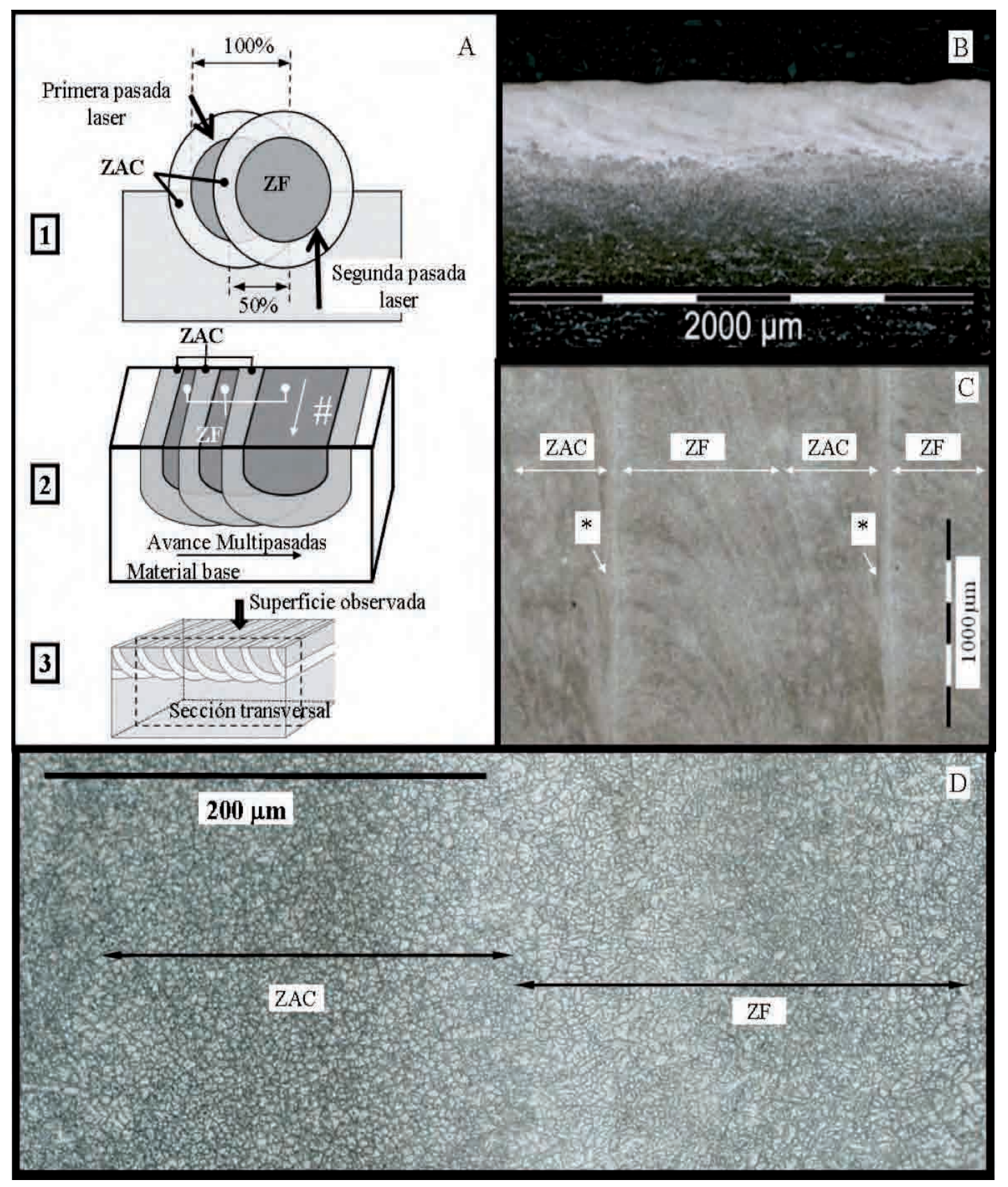

Figura 7. Efecto del tratamiento superficial de fusión por láser sobre la microestructura superficial (solapes al $50 \%$ ): A) Representación esquemática del sistema multipaso 1) cada paso cubre el $50 \%$ del paso siguiente; 2 ) esquema del efectos que produce el solapamiento sobre la superficie del material (\# representa el avance del haz del láser); 3) apariencia final del metal tratado. B) Micrografía óptica de la sección transversal (A3). C) Micrografía óptica de la superficie fundida con láser mostrada en A3 ( ${ }^{*}$ representa el límite de la zona fundida). D) Micrografía al MEB de detalle de la microestructura mostrada en $\mathrm{C}$.

Figure 7. Effect of the laser surface melting treatment on the surface microstructure (50\% overlap). A) Schematic representation of the multi-pass system: (1) each laser pass covers $50 \%$ of the previous pass; (2) schema of the effects of overlapping on the material surface (\# represents the advance of the laser beam); (3) final appearance of the treated material. B) Optical micrograph of the cross-section (A3). C) Optical micrograph of the laser melted surface shown in A3 ( ${ }^{*}$ represents the LMZ limit).D) SEM image of the microstructure in $C$. 


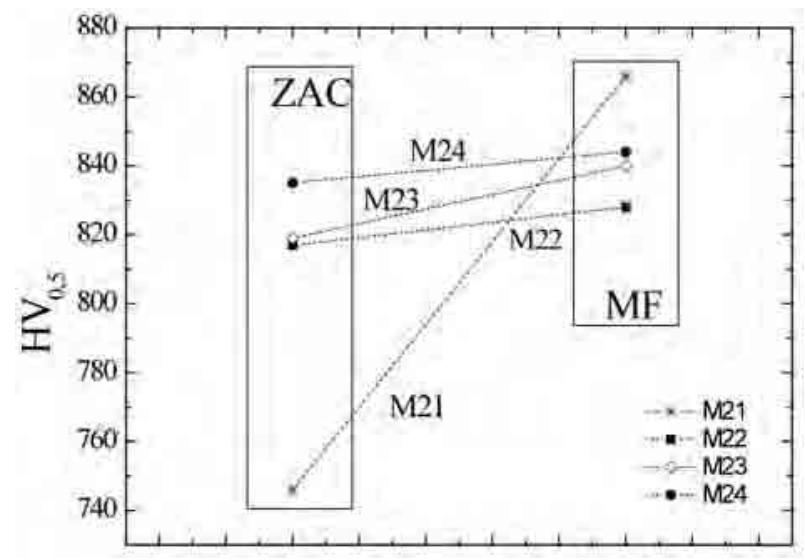

Figura 8. Valores medios de la microdurezas de la ZAC y MF obtenidos con los distintos parámetros de operación.

Figure 8. Average values of the $H A Z$ and $M Z$ microhardness obtained with the different operation parameters.

de dureza no es importante, manteniéndose, en todo caso, superior al material base $\left(760 \mathrm{HV}_{0,5} / 63\right.$ $\mathrm{HR}_{\mathrm{C}}$ ), siendo más importante la heterogeneidad cuando la densidad de potencia es elevada a bajas velocidades. El enfriamiento más lento de un baño fundido de mayor volumen (M21) determina que las zonas contiguas, en la superficie, estén más tiempo a temperatura elevada pudiendo producirse el revenido más arriba mencionado que, por otro lado, también se observa en la figura 6A. Menores densidades de potencia producen superficies más homogéneas.

La figura 9A muestra los patrones de difracción del acero AISI M2, antes y después de uno de los tratamientos de fusión por láser. En todos los casos estudiados (Tabla II), los patrones de difracción son similares. De su análisis queda constatada la presencia de austenita retenida en la superficie tratada con láser, como consecuencia del rápido enfriamiento del acero con alto contenido en carbono. La figura $9 \mathrm{~B}$ es una ampliación de la figura 9A, sobre la que se han identificado los carburos presentes en la superficie antes y después del tratamiento de fusión por láser. Los resultados de DRX muestran que, antes de fusión por láser, los tipos de carburos mayoritarios en el acero son del tipo MC (patrón estándar ICCD 1-1159) y $\mathrm{M}_{6} \mathrm{C}$ (patrón estándar ICCD 3-0980). Sin embargo, tras fusión por láser los picos que aparecen en el patrón de difracción corresponden a un carburo del tipo $\mathrm{MC}$ y otro tipo de carburo diferente del $\mathrm{M}_{6} \mathrm{C}$ (los picos no coinciden). Los espacios interlaminares, $d$, de esos picos corresponden al tipo de carburo hexagonal $\mathrm{M}_{2} \mathrm{C}$ con parámetros de red: $\mathrm{a}=2,9324 \AA$ y c $=4,6066 \AA$. Los resultados obtenidos, en este caso, concuerdan con los picos del patrón estándar 11-0680 del carburo hexagonal $\mathrm{Mo}_{2} \mathrm{C}$. La sustitución de algunos átomos de molibdeno por wolframio, hierro, cromo es responsable de una pequeña diferencia en los valores del espaciado interlaminar. La identificación realizada empleando DRX coincide con los resultados obtenidos en trabajos anteriores por microscopía, empleando técnicas ataque diferencial ${ }^{[22]}$.

Junto con la identificación de los carburos se ha obtenido la proporción de austenita retenida, en todos los casos. Los resultados de la tabla IV muestran como la cantidad de dicho constituyente varía según los parámetros de operación.

La retención de austenita a temperatura ambiente puede ser explicada debido a la más rápida cinética de solidificación de la fase $\gamma$ y a que esta reten-

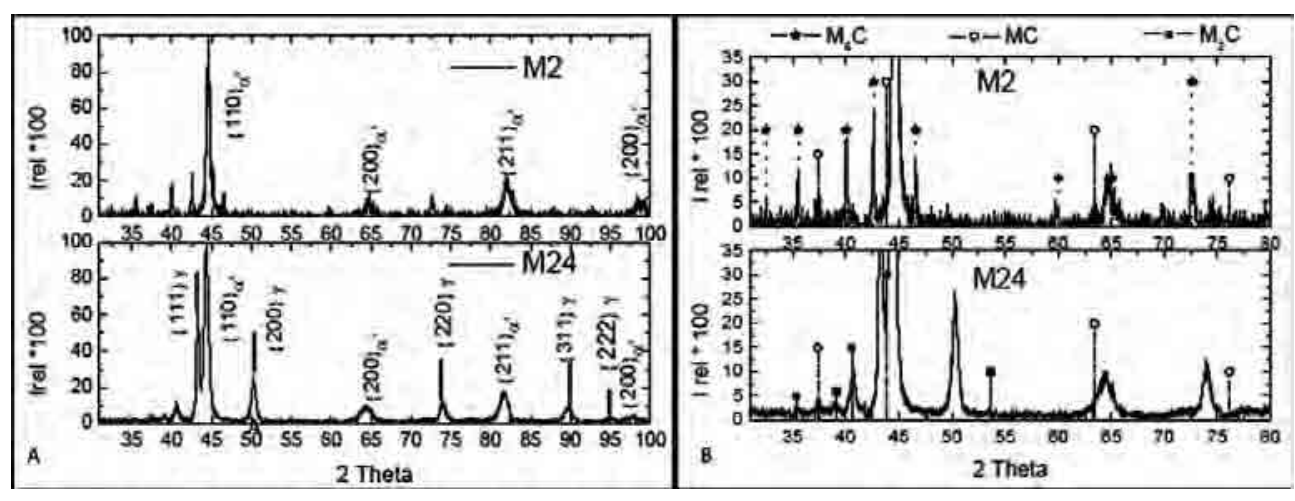

Figura 9. A) Patrones de DRX del acero AISI M2 antes y después del tratamiento de fusión superficial con láser. B) Detalle de A.

Figure 9. A) X-ray diffraction patterns for AISI M2 tool steel before and after laser-surface melting $B$ ) Detail of $A$. 
Tabla IV: Contenidos en austenita retenida y valores medios de microdureza obtenidos en superficie del acero AISI M2, tras tratamiento de fusión superficial por láser con los distintos parámetros de operación, M21, M22, M23 y M24.

Table IV: Quantities of retained austenita and average values of microhardness obtained in the surface of the AISI M2 steel after laser surface melting with the different operation parameters, M21, M22, M23, M24.

\begin{tabular}{lcccc}
\hline & M21 & M22 & M23 & M24 \\
\hline $\mathrm{Q}\left(\mathrm{W} / \mathrm{mm}^{2}\right)$ & 282,9 & 70,7 & 282,9 & 70,7 \\
$\mathrm{~V}(\mathrm{~mm} / \mathrm{s})$ & 25 & 25 & 60 & 60 \\
$\mathrm{~g}_{\text {retenida }}(\%)$ & 16 & 26 & 20 & 31 \\
$\mathrm{HV}_{0,5}$ & 806 & 823 & 830 & 840 \\
\hline
\end{tabular}

ción se vea favorecida durante el enfriamiento del baño solidificado. La velocidad de solidificación del baño fundido está relacionada con el volumen del baño fundido ${ }^{[3]}$ y éste, con la densidad de potencia. Cuanto menor es el valor de este parámetro menor es el volumen del baño fundido y mayor su velocidad de solidificación y enfriamiento. Está generalmente aceptado que cuanto mayor es la velocidad de solidificación y enfriamiento se favorecen, cinéticamente, la formación de austenita primaria a expensas de la formación de ferrita $\delta$ primaria y la formación de los carburos eutécticos $\mathrm{M}_{2} \mathrm{C}$ en lugar de los $\mathrm{M}_{6} \mathrm{C}^{[22]}$. Los resultados de DRX han confirmado este hecho, con la ausencia, en todos los casos, de carburos $\mathrm{M}_{6} \mathrm{C}$ en la superficie. Por lo tanto, se espera que al aumentar la velocidad de solidificación se produzca una transición del modo de solidificación de $\delta$ a $\gamma^{[17]}$. En consecuencia, se puede deducir que la velocidad de solidificación obtenida, en todos los casos, superó un valor umbral que ha dado lugar a la solidificación de austenita primaria.

Desde el punto de vista del enfriamiento del baño solidificado, si bien es conocido que a mayores velocidades de enfriamiento aumenta la temperatura Ms, conduciendo a una disminución de la fracción de volumen de austenita retenida ${ }^{[24}$ y 25$]$, otros factores como son la presencia de elementos de aleación en la austenita y el tamaño de grano austenítico pueden modificar esta regla.

Así, está demostrado que los elementos de aleación disueltos en la austenita la hacen más estable y, por lo tanto, disminuye la temperatura Ms. También, se ha comprobado que al reducir el tamaño de grano austenítico disminuye Ms, por lo tanto, el afino de la estructura dendrítica de la austenita producida por el aumento de la velocidad de solidificación y enfriamiento de la superficie refundida favorece la presencia de austenita retenida ${ }^{[24]}$.

Al emplear menor densidad de potencia para la misma velocidad del haz del láser se produce un menor baño fundido que enfría más rápido conduciendo a un afino del grano austenítico que disminuye Ms y, por tanto, aumenta la proporción de austenita retenida. Este hecho, como ya se ha mencionado en la introducción, ya había sido demostrado por otros autores $^{[18 \text { y 19] }}$ para aceros de herramientas con contenidos en carbono menores que este caso. Los resultados muestran que el aumento de la velocidad de barrido del haz a potencia constante también produce el mismo efecto. Los menores valores de austenita retenida se registran a densidades de potencia altas y bajas velocidades, por lo que modificando estos parámetros podríamos conseguir reducir esta cantidad al mínimo, sin embargo, como hemos visto anteriormente, las altas densidades de potencia de operación producen mayor heterogeneidad superficial, por lo cual parece más conveniente reducir la velocidad con el fin de disminuir la presencia de austenita retenida.

La tabla IV recoge los valores medios de la microdureza en la superficie del material tras fusión por láser. En todos los casos, es superior al material base $\left(760 \mathrm{HV}_{0,5} / 63 \mathrm{HR}_{\mathrm{C}}\right)$. La figura10 muestra que este

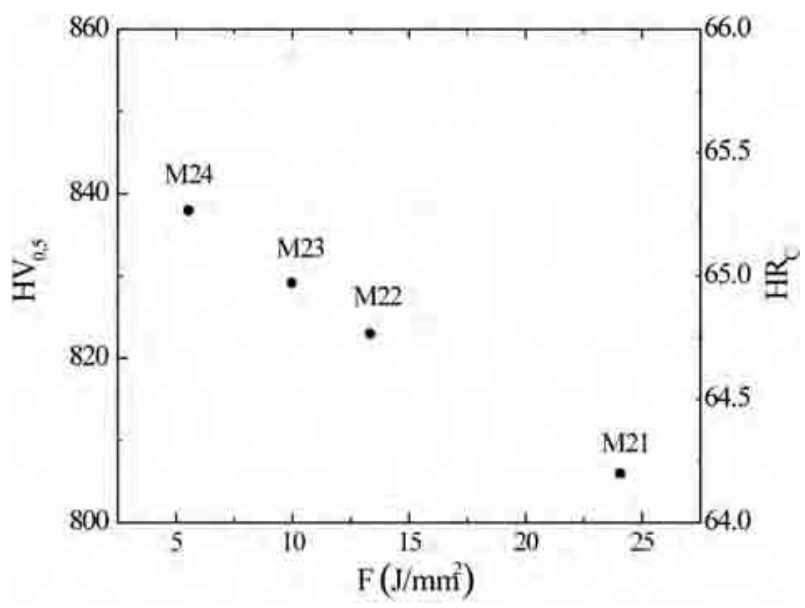

Figura 10. Variación de la microdureza en la superficie del material tras fusión superficial con láser en función de la energía aportada por el haz láser por unidad de superficie. (Equivalencia $\mathrm{HR}_{\mathrm{C}}$ ).

Figure 10. Changes of microhardness with the input energy of laser beam in laser melted surface. (HR $\mathrm{R}_{C}$ equivalence). 
valor depende de los parámetros de operación: así, cuando la energía aportada disminuye, la dureza aumenta y, aunque el contenido en austenita retenida sea elevado, parece que el efecto se ve compensado con el menor tamaño de grano de la microestructura y el mayor número de dislocaciones en la martensita de elevada dureza, cuando la velocidad de enfriamiento del acero fundido con láser es alta. Esto, sucede a bajas densidades de potencia y velocidad de haz de láser elevada.

En cuanto a la resistencia al desgaste, se puede decir que el material tratado superficialmente por fusión con láser presenta un mejor comportamiento que el material base, según se desprende de los resultados que se muestran en la figura 11. Esto, de acuerdo con los valores de dureza obtenidos. El estudio del fondo de huella nos muestra que el tipo de desgaste producido en el material antes y después del tratamiento superficial por láser es distinto[ ${ }^{[26]}$. Las partículas masivas de carburos serán las responsables de un peor comportamiento del acero convencional, como se muestra en la figura 12A, en la que se puede ver cómo el arranque de material en la banda de carburos gruesos paralelos a la dirección de forja ha producido surcos gruesos propios de desgaste abrasivo. Mientras que, en el fondo de las huellas de desgaste, en el caso del material tratado con fusión por láser, se observan surcos suaves en la dirección de deslizamiento del pin con una cierta deformación plástica además de partículas de alúmina transferidas del pin, hechos que puede ser considerados más propios de un desgaste adhesivo. En ninguna zona del fondo de la huella de la superficie fundida con láser, se ha podido observar

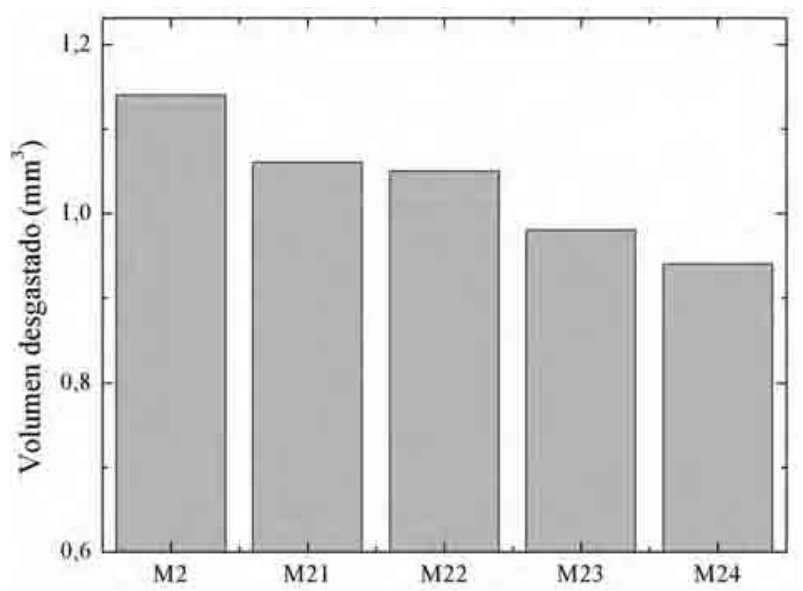

Figura 11. Resistencia al desgaste por deslizamiento: metal base y las superficie fundida por láser-M21, M22, M23, M24-.

Figure 11. Wear resistance by sliding: base metal and laser melted surfaces -M21, M22, M23, M24-.

la presencia de un mecanismo de desgaste "abrasivo". Esto ha sido gracias a la optimización de la distribución de carburos conseguida durante la fusión superficial. Además, parece que el contenido en austenita retenida no afecta a los resultados de desgaste, manteniéndose más resistentes las superficies tratadas con mayor dureza. El tratamiento que se realiza con menor aporte de energía el que presenta un mejor comportamiento a desgaste.

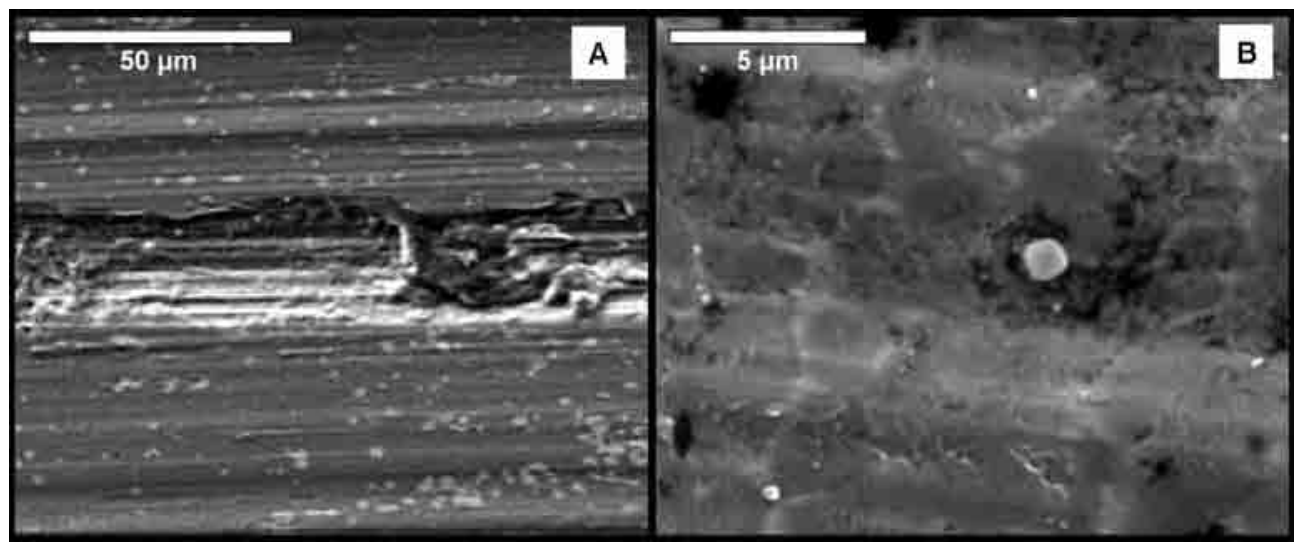

Figura 12. Micrografías al MEB del fondo de huella de desgaste. A) AISI M2. B) Superficie fundida con láser del acero AISI M2 (partícula blanca redonda: restos de alúmina -análisis EDAX-).

Figure 12: SEM images of the wear track by sliding on A) AISI M2. B) Laser melted surface of AISI M2 steel (round white particle: alumina rest-EDAX analysis-). 


\section{CONCLUSIONES}

De acuerdo con los resultados presentados, se pueden realizar las siguientes conclusiones:

- Todos los parámetros de operación del láser utilizados en este trabajo son adecuados para obtener una superficie con un relativamente buen acabado y libre de defectos.

- La variación de dichos parámetros influye en la morfología de los cordones: al aumentar la energía aportada por unidad de superficie aumenta el área superficial afectada y la profundidad total del cordón.

- La profundidad y microestructura de la ZPF depende de la distribución y tamaño de carburos en el material base, además de los parámetros de operación.

- Los carburos masivos del material base se disolvieron y no se observan en la superficie solidificada. La microestructura de la zona fundida está constituida por finas dendritas de martensita rodeadas de una eutéctica de martensita y carbu$\operatorname{ros} \mathrm{M}_{2} \mathrm{C}$ y $\mathrm{MC}$ de pequeño tamaño. Se ha confirmado la presencia de austenita retenida en la zona fundida.

- El tratamiento de fusión superficial multipasada con solapamiento necesario para cubrir un área de la herramienta produce una superficie en la que se puede distinguir, en cada cordón producido por el paso del haz del láser, dos zonas: ZF y ZAC. Ambas presentan una microestructura similar y la variación de dureza es pequeña. Esta homogeneidad se ve afectada por valores de la densidad de potencia altos.

- El contenido en austenita retenida depende de los parámetros de operación, obteniendo los menores valores a altas densidades de potencia y bajas velocidades del haz de láser. Sin embargo, se ha demostrado que estas condiciones conducen a superficies más heterogéneas que pueden presentar un peor comportamiento en servicio.

- La estructura obtenida en la superficie, después de la fusión con láser, tiene un alto nivel de dureza. Esta dureza es tanto más elevada cuando menor es la energía aportada por el haz. El pequeño tamaño de grano obtenido con bajas densidades de potencia y altas velocidades del haz del láser compensa el ablandamiento producido por los mayores valores de austenita retenida que se obtienen en estos casos.

- La superficie, después de la fusión con láser, muestra una mejor resistencia al desgaste que el material base. Es consecuencia de su mayor dureza y de la mejor distribución de los carburos.

\section{Agradecimientos}

Este trabajo pudo ser realizado gracias a la financiación concedida por la Dirección Xeral de I+D+i de la Xunta de Galicia al proyecto $\mathrm{n}^{\circ}$ PGIDIT06TMT00402CT.

\section{REFERENCIAS}

[1] G. Roberts, G. K. Krauss y R. Kennedy, Tool Steels, ASM International, EEUU, 1998, pp. 251-289.

[2] Tool Materials, ASM International, EEUU, 1995, pp. 119-153.

[3] W. M. Steen, Laser Materials Processing, Springer Verlag, Berlin, Alemania, 1998, pp. 435-543.

[4] G. Muñiz, A. Conde, B. J. Fernández, R. Varela, I. Garcia y J. De Damborenea, Rev. Metal. Madrid 39 (2003) 443-451.

[5] S. Kac y J. Kusinski, Surf. Coat. Technol. 180181 (2004) 611-615.

[6] C. Navas, A. Conde, B. J. Fernández, F. Zubiri y J. de Damborenea, Surf. Coat. Technol. 194 (2005) 136-142.

[7] W. Darmawan, J. Quesada y R. Marchal, Surf. Eng. 23 (2007) 112-119.

[8] R. Colaco, C. Pina y R. Vilar, Scr. Mater. 41 (1999) 715-721.

[9] C. T. Kwok, F. T. Cheng y H. C. Man, Surf. Coat. Technol. 202 (2007) 336-348.

[10] S. Kac, J. Kusinski, A. Zielinska-Lipiec y I. Wronska, J. Microsc. Oxford 224 (2006) 65-67.

[11] M. Boccalini y H. Goldenstein, Int. Mater. Rev. 46 (2001) 92-115.

[12] S. Kac y J. Kusinski, Mater. Chem. Phys. 81 (2003) 510-512.

[13] J. Kusinski, Appl. Surf. Sci. 86 (1995) 317-322.

[14] J. Kusinski, Metall. Trans. A 19 (1988) 377 382.

[15] L. Ahman, Metall. Trans. A 15 (1984) 1.8291.835 .

[16] K. Y. Benyounis, O.M. Fakron, J.H. Abboud, Mater. Design 30 (2009) 674-678

[17] G. Hoyle, High Speed Steels, Butterworths, London, UK, 1988, pp 10-24.

[18] R. Colaco y R. Vilar, J. Mater. Sci. Lett. 17 (1998) 563-567.

[19] R. Colaço y R. Vilar, Mater. Sci. Eng. A 385 (2004) 123-127.

[20] R. Colaco y R. Vilar, Rev. Metal. Madrid 34 (1998) 135-139.

[21] R. Colaco, E. Gordo, E. M. Ruiz-Navas, M. Otasevic y R. Vilar, Wear 260 (2006) 949-956. 
[22] J. Arias, M. Cabeza, G. Castro, I. Feijoo, P. Merino y G. Pena, X Congreso Nacional de Materiales, San Sebastián, España, 2008, Universidad de Mondragón (Eds.), San Sebastián, España, 2008, pp. 101-104.

[23] H. Fredriksson y S. Brising, Scand. J. Metall. 5 (1976) 268-275.

[24] J.A. Pero-Sanz, Aceros, Ed. Dossat, Madrid, España, 2004, pp. 64-81.
[25] D. H. Bhadeshia y R. Honeycombe, Steels: Microstructure and Properties, Butterworths, Oxford, Inglaterra, 2006, pp. 95-120.

[26] G. W. Stachowiak, A. W. Batchelor y G. B. Stachowiak, Experimental Methods in Tribology. Elsevier, Amsterdam, Holanda, 2004, pp. 170 179. 